\title{
Reproduktionsmedizin
}

\section{Samenspender bleiben nicht mehr anonym}

\author{
Seit 2007 schreibt deutsches und EU-Recht vor, dass die Daten von \\ Samenspendern 30 Jahre lang aufgehoben und rückverfolgbar sein \\ müssen. Anonymität war den Samenspendern sicher, auch davor \\ schon. Jetzt gibt es ein aktuelles Urteil, dass die Schweigepflicht des \\ Arztes aushebelt.
}

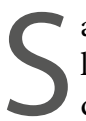
amenspender haben auch in Altfällen vor 2007 keinen Anspruch auf die ihnen zugesicherte Anonymität. Das Recht des Kindes an der Kenntnis seiner Herkunft steht höher, wie jetzt das Oberlandesgericht (OLG) Hamm entschied (I-14 U 7/12). Der Arzt verstoße daher nicht gegen seine Schweigepflicht, wenn er die Daten herausgibt. Damit gab das OLG der im März 1991 in einem Institut eines Arztes in Essen durch heterologe Insemination gezeugten Sarah P. recht. Von dem Arzt wollte Sarah nun wissen, von welchem Mann sie abstammt. Der Arzt lehnte dies ab. Er habe den Samenspendern Anonymität zugesichert und dies entsprechend mit der Mutter vereinbart. Dieses „Geheimhaltungsinteresse“ sei höher zu bewerten, als das Auskunftsinteresse des Kindes.

\section{Menschenwürde vor Geheimhaltung}

Das OLG Hamm gewichtete die Interessen nun aber anders herum: Das Interesse der Klägerin, ihre Abstammung zu erfahren, sei höher zu bewerten als die Interessen des Arztes und der Samenspender an einer Geheimhaltung. Mögliche gegenläufige Interessen der Mutter und des gesetzlichen Vaters seien hier unerheblich, weil beide mit der Auskunftserteilung an die Klägerin einverstanden sind. Zur Begründung verwies das OLG auf die allgemeine Menschenwürde und das Recht auf freie Entfaltung der Persönlichkeit. Die Abstammung spiele eine große Rolle, um die „Persönlichkeit verstehen und entfalten zu können“. Hinter diese „fundamentale Rechtsposition" müssten die Berufsfreiheit des Arztes und auch das Persön-

\section{Samenspenden in Deutschland}

Seit 1986 ist in Deutschland die Spermaspende eine legale Behandlungsmethode der künstlichen Befruchtung. Etwa 100.000 Menschen sollen hierzulande leben, die mittels einer Samenspende gezeugt wurden.

In Anspruch nehmen dürfen eine Samenspende heute verheiratete und unverheiratete Paare. Einige Bundesländer verlangen allerdings die Vorlage eines notariell beglaubigten Vertrages, in dem die gegenseitige finanzielle Absicherung festgeschrieben ist. Samenspenden an Singles oder gleichgeschlechtliche Paare gelten als rechtlicher Graubereich.

Zu den Persönlichkeitsrechten eines Menschen gehört es, seine genetische Herkunft zu kennen, das hat das Bundesverfassungsgericht bereits 1989 entschieden. Bislang gibt es in Deutschland allerdings keine detaillierten Vorschriften dafür, wie betroffene Kinder ihren Rechtsanspruch im konkreten Fall durchsetzen können.

Nachwuchs aus der Samenbank. Anonymität kann Spendern nun nicht mehr zugesichert werden.

lichkeitsrecht des auf seine Anonymität vertrauenden Spenders zurücktreten, urteilte das OLG. Beide seien „nicht in ihren zentralen Bereichen betroffen “ und schon deswegen weniger schutzbedürftig, weil sie mit der Möglichkeit hätten rechnen müssen, dass das gezeugte Kind seinen Vater kennen will. Dass im konkreten Fall der Arzt die Auskunft nicht mehr geben kann, habe er noch nicht ausreichend belegt. Unmöglich sei die Auskunft erst, wenn er die Informationen, ,auch nach einer umfassenden Recherche in seiner Praxis" nicht mehr beschaffen könne. Das OLG ließ keine Revision zu, dagegen kann der Arzt aber Beschwerde beim Bundesgerichtshof einlegen.

\section{Recht auf Kenntnis der eigenen genetischen Herkunft}

Seit 2007 schreibt deutsches und EURecht vor, dass die Daten von Samenspendern 30 Jahre lang aufgehoben und rückverfolgbar sein müssen. Betroffen von dem Urteil sind vor allem Kinder, die vorher gezeugt wurden. Auch zuvor sei dies aber ähnlich in den meisten Berufsordnungen der Landesärztekammern festgeschrieben gewesen, sagte Najib Nassar, Sprecher und Vorstandsmitglied des Bundesverbandes Reproduktionsmedizinischer Zentren in Deutschland. Schon 1989 hatte das Bundesverfassungsgericht das Recht auf Kenntnis der eigenen genetischen Herkunft hervorgehoben.

Helmut Laschet 